\title{
PANTIR - A DUAL CAMERA SETUP FOR PRECISE GEOREFERENCING AND MOSAICING OF THERMAL AERIAL IMAGES
}

\author{
I.Weber $^{\text {a,* }}$, A.Jenal ${ }^{\mathrm{a}}$, C.Kneer ${ }^{\mathrm{b}}$, J.Bongartz $^{\mathrm{a}, \mathrm{b}}$ \\ ${ }^{a}$ Fraunhofer FHR, 53424 Remagen, Germany - (immanuel.weber, alexander.jenal, jens.bongartz)@fhr.fraunhofer.de \\ ${ }^{\mathrm{b}}$ University of Applied Sciences Koblenz, 53424 Remagen, Germany - (kneer, bongartz)@hs-koblenz.de
}

KEY WORDS: aerial, thermal imaging, image fusion, multispectral

\begin{abstract}
:
Research and monitoring in fields like hydrology and agriculture are applications of airborne thermal infrared (TIR) cameras, which suffer from low spatial resolution and low quality lenses. Common ground control points (GCPs), lacking thermal activity and being relatively small in size, cannot be used in TIR images. Precise georeferencing and mosaicing however is necessary for data analysis. Adding a high resolution visible light camera (VIS) with a high quality lens very close to the TIR camera, in the same stabilized rig, allows us to do accurate geoprocessing with standard GCPs after fusing both images (VIS+TIR) using standard image registration methods.
\end{abstract}

\section{INTRODUCTION}

\subsection{Motivation}

In fields like hydrological and agricultural research or monitoring, but also in surveying of industrial or urban facilities, measurements of temperature distributions of spacious areas are of interest. This is a common airborne remote sensing application for thermal infrared (TIR or longwave infrared LWIR) cameras, like microbolometer sensors. To this day the spatial resolution of these sensors is limited and far away from cameras working in the visible range of light (VIS). Even expensive high-end imagers have resolutions not much larger than $640 \times 480$ pixels. In addition lens materials, which must be used in LWIR applications, are very difficult to produce and to handle, hence precise high quality lenses with short focal lengths are either very expensive or even not achievable. These two issues result in low spatial resolution on the ground and due to the long focal lengths either higher flying altitudes or narrow swath widths. Furthermore lens distortions can be a serious problem. These issues alone would make precise georeferencing and automated mosaicing using structure from motion (SfM) methods (Wu, 2013) of thermal images difficult, as ground control points (GCPs) have to be large in size and number due to the small covered area of each image. But the characteristics of thermal images are making these tasks even harder. Besides of hot spots or significant temperature differences thermal images are not rich in contrast and mostly homogeneous depending on the composition of the scenery. Due to these disadvantages it is nearly impossible to identify GCPs laid-out for VIS cameras in TIR images. Even existing (manmade or naturally occurring) GCPs with a thermal signature rarely exist in a sufficient number, distribution and size for aerial surveys. Detecting such points aside from urban areas is a non-trivial task. However without accurate GCPs a precise georeferencing of the images is impossible. Mosaicing thermal aerial images with SfM algorithms to larger heat maps is also difficult because of the lack of well distributed structures, respectively features, in the images.

\subsection{Related Work}

On-board thermal infrared cameras are usually coupled to high precision GPS/IMU devices (Mostafa et. al., 2001). The airborne GPS/IMU enables direct georeferencing of the resulting thermal images using exterior orientations (latitude, longitude, altitude, roll, pitch, and yaw). Suitable photogrammetry software converts the collected data into orthorectified thermal image mosaics without reference to ground features. Additionally the data-processing workflow includes corrections of atmospheric effects and lens distortions. One drawback of this established approach is the comparatively high cost of a high precision IMU device in the range $>100.000$ EUR. An approach using an inexpensive IMU is described in Miraliakbari et al. (2010).

\subsection{Concept}

To reduce operational costs we developed a setup that does not rely on IMU data and utilizes moderately priced SfM software. Our targeted areas of applications are measuring the surface temperature of rivers and lakes in hydrology and monitoring the temperature of leaves, plants or canopies as an indicator of leaf transpiration rate and hence of plant stress.

Our approach to introduce SfM algorithms to aerial thermal imaging is to add a second modality beside the thermal camera. One modality where the issues of low spatial resolution and low image contrast are not playing a significant role, namely the mentioned VIS cameras. That kind of camera is off-the-shelf available at a low price point with high resolution in the mega pixel range. High quality lenses in that wavelength range are also obtainable without difficulties. Furthermore VIS images are mostly rich in contrast, as more structural information is carried in the visible range. In addition the sheer amount of pixels leads to better contrast. Data resulting from these cameras can easily be fed into automated georectification and geomosaicing tools. For precise georeferencing these tools allow manual registration of pre-measured GCPs. When the resulting images show the same scene as the one captured with

\footnotetext{
* Corresponding author
} 
the thermal camera and the footprints match, image pairs can be fused to a two-layer image. Two achieve that both cameras have the same field of view, one would ideally use an optical setup where a beam splitter would image the scene onto both camera sensors. However as mentioned earlier optical materials for VIS and TIR imaging greatly differ and the latter are very difficult to handle, hence the construction of such a setup would be (nearly) impossible. Another approach suitable in remote sensing applications is to put both cameras as close together as possible, so that the distance of their optical axes becomes smaller than a pixel's ground footprint. That limits the minimum flying altitude, but simplifies the physical setup. Nevertheless the construction needs to align the optical axes as parallel as possible, hence requiring great mechanical precision, in order to prevent relative rotation and skewing of the images. To account for that a small toolchain of image processing steps should be used, which uses standard image registration methods to determine remaining necessary image transformations, cut the images to correct sizes and to fuse both into one two-layer image. In any subsequent steps the VIS layer can be used for registration, georectification and geomosaicing. As the standard tools support multilayer images, the TIR layer is transformed alongside, resulting in precise georeferenced data.

\section{METHOD}

\subsection{Technical Aspects and Construction}

The essential parts of our system are a TIR camera (Allied Vision Pearleye P-030) and a panchromatic (PAN) VIS camera (Allied Vision Mako G-419b). The focal length of the lens of the visible camera is adjusted so that its field of view matches the one of the thermal camera as closely as possible. A custom designed, lightweight aluminium rack precisely aligns both cameras to each other and keeps them stable in flight (figure 1). The rack consists of a solid mount which holds the camera and a housing which covers the camera bodies, to prevent damage while take-off and landing, it also reduces the wind load while still allowing airflow to cool the cameras. The rack is mounted in an active, electromechanical, two axis stabilization system (gimbal) which guarantees a nadir viewing angle of the sensors during image acquisition, regardless of aircraft motion (figure 1). The gimbal itself is mounted outside of the aircraft. The cameras are connected with a rugged embedded PC via Ethernet, which is positioned inside of the aircraft. In addition both cameras are connected to each other, to allow hardware synchronization. Cameras, gimbal and embedded PC are powered by a specifically designed power supply that allows using the aircraft power in flight and a separate battery on the ground, to spare the aircraft's own. To further reduce wind load a wind deflector is positioned in front of the gimbal. A small device, the so-called SensorBay, which can capture various environmental parameters like outside air temperature and outside air humidity, supplements the system. It can also capture the absolute altitude above ground provided by an external radar altimeter. The complete system weights less than $10 \mathrm{~kg}$.

\subsection{Acquisition}

The embedded PC runs a tailor made acquisition application which is based on the modular AMLS software framework AWS. It connects both cameras, the SensorBay, the aircraft GNS system and the flight management system, which provides a trigger signal. Its task is to acquire images of both cameras on trigger action, simultaneously capture all SensorBay data, the position and attitude date from the GNSS, synchronize that data and store it on the internal SSD. For start-up and setup of the system the application provides a GUI which allows to see the camera images and all other data and enables the user to set all mission specific parameters. A second GUI allows the pilot to monitor the system status during flight.

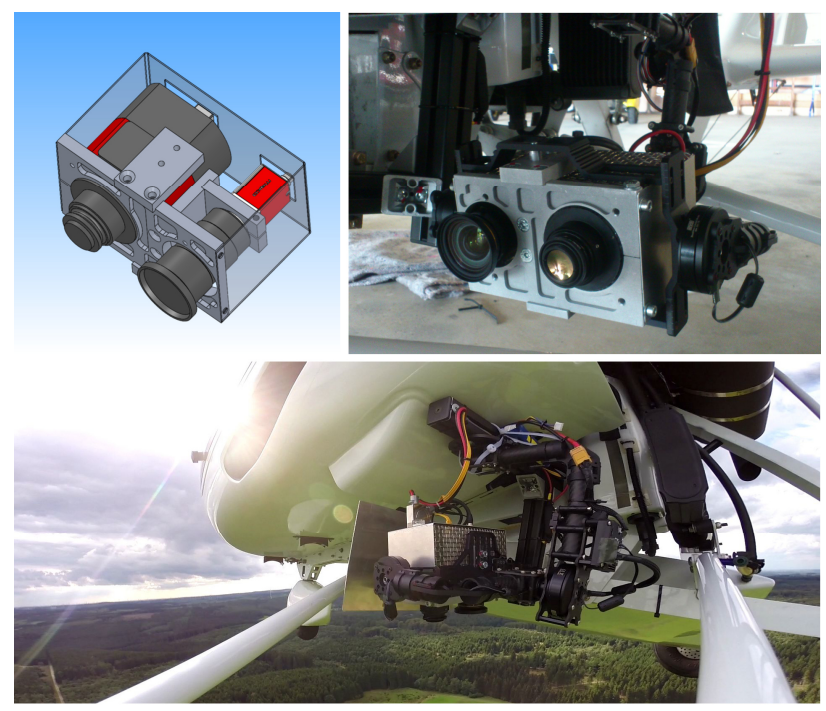

Figure 1. Top left: CAD drawing of the PanTIR system, consisting of a panchromatic (right) and a TIR camera (left). Top right: PanTIR mounted on a stabilized gimbal attached to an aircraft. Bottom: PanTIR-system airborne pointing nadir to the ground.

\subsection{Processing and Registration}

As above-mentioned after doing a survey the image data needs to be preprocessed before it can be fed into SfM applications. Due to the difficult to handle optical materials for LWIR cameras lenses with short focal lengths often show significant lens distortion effects. These effects need to be corrected before any other processing step can be applied. Our camera shows a typical barrel distortion. Other forms like tangential distortion are not playing a significant role, hence we only correct the radial distortion. Standard tools, like printed checkerboards, can usually not be used due to the non-discriminability of black and white paper in thermal images. At the moment images of a building are used, where many straight lines are visible in the thermal image. Based on these images we compute the coefficients necessary to correct the distortion (Zhang, 2000). Then we can apply the correction on all thermal images of the survey. As a side note, the optical quality of the visible cameras lens is very good, hence no visible distortion occurs and hence no correction of these images is necessary. After the distortion correction both images need to be registered to each other to enable the fusion into one two-layer image. The registration allows the computation of a transformation, consisting of a translation, rotation, scaling and in worse cases skewing, which lets us transform the thermal image into the coordinate system of the panchromatic image. As the cameras are precisely aligned to each other the optical axes are almost parallel, hence the occurring transformations are small. One needs to be aware that the transformations are dependent on the flying height, as a larger altitude will increase the effects of non-parallel optical axes. However scaling and rotation are constant with respect to altitude. The precision of the mechanical alignment is good enough to ignore skewing. To register the images an approach based on manual control points is used, as automated SIFT 
methods (Lowe, 2004) tend not to create usable results, due to the different characteristics of thermal and visible images. As the principal idea is based on the fact, that with sufficient altitude the distance between the cameras, will become smaller as one pixel's footprint on ground, the minimal altitude was determined beforehand, which is in our system $200 \mathrm{~m}$. We took pairs of images from the desired flying altitudes, which show scenes with a significant amount of thermal and visible features. Then points showing the same feature in each image are measured and fed into algorithms of Mathworks' MATLAB Image Processing Toolbox to compute the transformation. The points should be distributed over the image and their number should be way larger than necessary, to overdetermine the intrinsic systems of equations and hence increase robustness with respect to user selection errors and increase the overall quality of the results. The resulting transformations can then be applied to the distortion corrected thermal images. Now each pair of thermal and visible image can be fused into one twolayer image. The final step is to cut the image. As the footprint of the visible image is a little bit larger than the thermal one, the non-overlapping areas are cut off. The result is a fully registered two-layer image consisting of a high resolution visible image and in comparison mediocre resolution thermal image. These images can be fed into SfM tools. They can use the high resolution visible layer to find features, register the images, compute point clouds and create georeferenced orthomosaics. The intrinsic transformations will be automatically applied to the thermal layer.

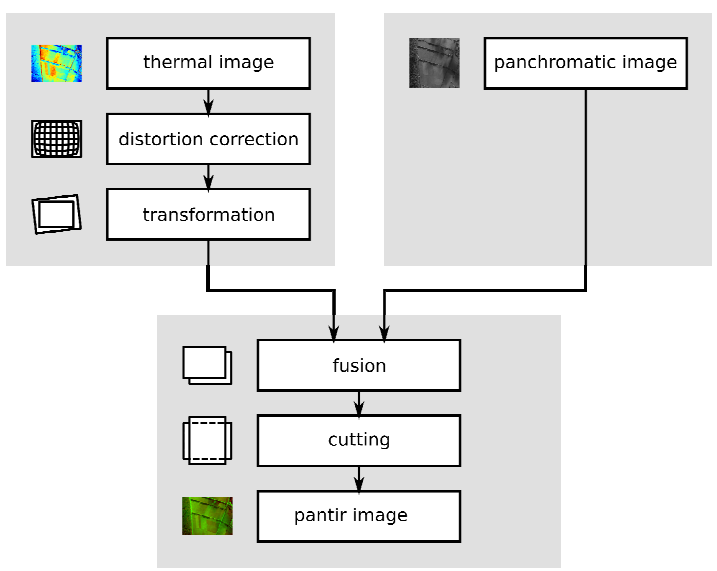

Figure 2. Flowchart that summarizes the preproccesing steps.

\section{EXPERIMENTS}

\subsection{Data}

The sensor solution was tested during several flights, in advance of a larger surveying campaign in the field of hydrology. The data presented here belongs to a selected region next to the airfield Dahlemer Binz in Germany, where the aircraft of the authors is stationed. This region (Steinbruch, figure 3) contains a stone pit which has some larger dumps of waste material, its size is roughly $6.5 \mathrm{sqkm}$. In accordance with the planned hydrology project the average flying altitude was around $3000 \mathrm{ft}$. During an acquisition time of 12 minutes 93 images were captured.

\subsection{Results}

At flight altitudes of $3000 \mathrm{ft}$ the swath widths is $825 \mathrm{~m}$ resulting in resolutions of $0.4 \mathrm{~m}$ for the PAN VIS camera and $1.3 \mathrm{~m}$ for the TIR camera. The image data could be fused according to the implemented algorithm described above. The mosaicing was possible as the panchromatic images carry a large amount of structures and hence good features in large number could be extracted and used in the SfM toolchain. Georeferencing was also possible as GCPs of an appropriate size can easily be recognized. The results show that our approach solves the initially mentioned issues in a way that the resulting images fulfil the requirements for further field specific analysis. Hence thermal aerial surveys can be easier conducted as standard GCPs can be used, which do not have to be thermally active and huge in size.

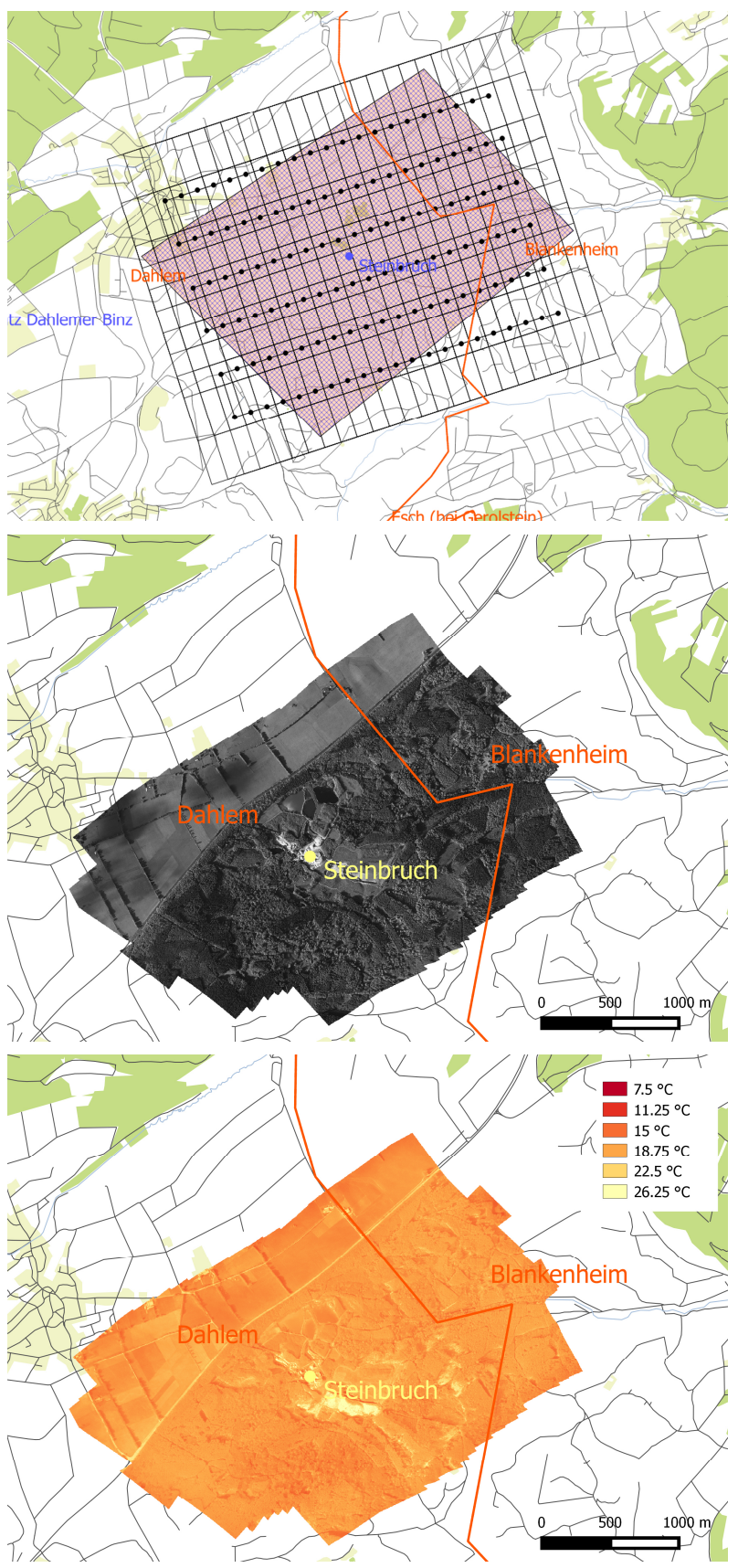

Figure 3. Top: Flightplan of the surveyed region (Steinbruch). Middle: Georeferenced orthomosaic of the VIS camera using SfM algorithm. Bottom: Visualization of TIR image layer. 


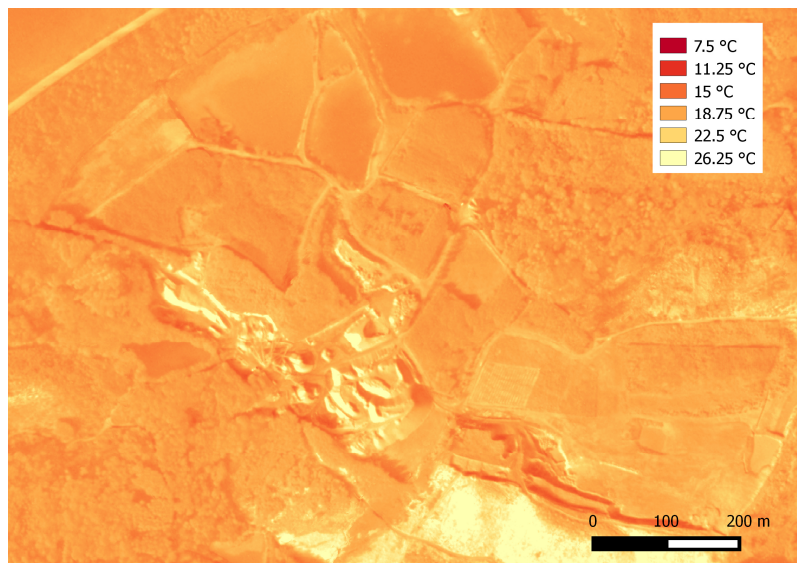

Figure 4. Magnification of the TIR image to show the stone pit in more detail.

\section{CONCLUSION AND FUTURE WORK}

The results show that by using an additional panchromatic camera it is possible to make thermal imaging feasible for SfM algorithm for mosaicing and georeferencing of aerial images. Further work is necessary to quantify the results exactly and to compare the results with traditional GPS/IMU solutions.

The approach can be extended to other wavelength ranges - like NIR or SWIR - as a starting point for multispectral imaging systems. Furthermore the number of cameras can be increased. At the moment of writing this paper a four camera system for VIS/NIR applications is under development, which can be seen in figure 5 . The system consists of one panchromatic camera for the reference aerial imaging and three spectral channels in the VIS/NIR range using appropriate bandwidth filters. Final goal is to achieve a setup of six cameras.
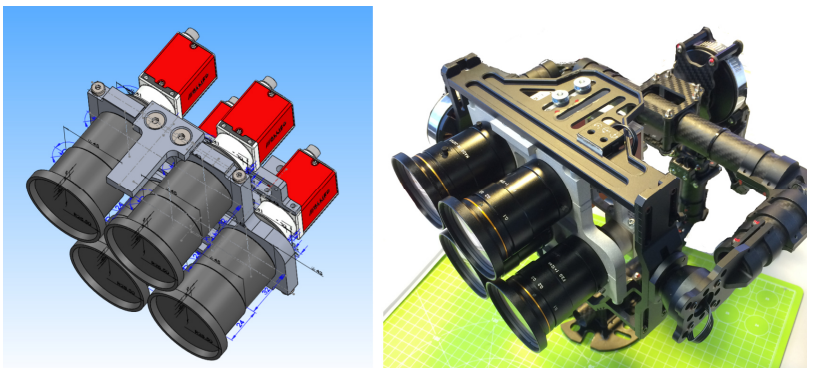

Figure 5. A four-camera system is under development. The system consists of one panchromatic camera for the reference aerial imaging and three spectral channels in the VIS/NIR range using appropriate bandwidth filters.

Due to the fact that the form factors of VIS/NIR, SWIR and TIR cameras are converging rapidly, a versatile system rack will be available in the near future which covers the whole optical spectrum from VIS to LWIR. The system is small in size, lightweight, stabilized and easy to handle, so adapting it to various airborne platforms should not be difficult. Furthermore automatic lens correction routines and atmospheric corrections will be integrated in the software framework of the multicamera setup.

\section{ACKNOWLEDGEMENTS}

The authors want to thank Allied Vision Technologies GmbH for kindly providing a Pearleye P-030 camera for the experiments free of charge.

\section{REFERENCES}

Lowe, D.G., 2004. Distinctive Image Features from ScaleInvariant Keypoints. International Journal of Computer Vision, 60, 2, pp. 91-110.

Miraliakbari, A., Hahn, M. and Engels, J., 2010. Development of a Low-cost Sensor System for Use on Gyrocopters. Canadian Geomatics Conference 2010 and ISPRS Com. I Symposium, International Archives of the Photogrammetry, Remote Sensing and Spatial Information Sciences, Vol. XXXVIII, Part 1, 7 pages.

Mostafa, M. and Hutton J., 2001. Direct positioning and orientation systems. How do they work? What is the attainable accuracy? APPLANIX Corporation, 85 Leek Cr., Richmond Hill Ontario, Canada L4B 3B3.

Wu, C., 2013. Towards Linear-Time Incremental Structure from Motion, 3D Vision - 3DV 2013, 2013 International Conference on 3D Vision, pp.127-134.

Zhang, Z., 2000. A flexible new technique for camera calibration. IEEE Transactions on Pattern Analysis and Machine Intelligence,

22(11): pp. 1330-1334. 